\title{
PERSPECTIVAS DA QUÍMICA MEDICINAL PARA O SÉCULO XXI: DESAFIOS E OPORTUNIDADES
}

\author{
Carolina Horta Andrade, ${ }^{a}$ Arthur E. Kümmerle ${ }^{\mathrm{b}}$ e Rafael V. C. Guido ${ }^{\mathrm{c}, *}$ \\ ${ }^{a}$ Faculdade de Farmácia, Universidade Federal de Goiás, Rua 240, Qd. 87, St. Leste Universitário, 74605-170 Goiânia - GO, Brasil \\ bDepartamento de Química, Universidade Federal Rural do Rio de Janeiro, 23897-000 Seropedica - RJ, Brasil \\ 'Instituto de Física de São Carlos, Universidade de São Paulo, Av. Joao Dagnone, 1100 - Jardim Santa Angelina, 13563-120 São \\ Carlos - SP, Brasil
}

Recebido em 18/09/2017; aceito em 06/12/2017; publicado na web em 18/01/2018

\begin{abstract}
MEDICINAL CHEMISTRY PERSPECTIVES FOR THE 21ST CENTURY: CHALLENGES AND OPPROTUNITIES. In the 21 st century, medicinal chemists will face many challenges to improve the quality of life of populations. The challenges consist of emerging infectious (ex. bacterial, viral and parasite infections) and non-communicable diseases (ex. autoimmune, Alzheimer disease, Parkinson's disease) that will require innovative technologies (ex. microfluidics, nanotechnology, biotechnology) to be fully understood and combated. In this work, we indicate trends, perspectives and opportunities related to drug discovery as well as highlight the tools and strategies that could be used in drug discovery of the 21st century.
\end{abstract}

Keywords: Medicinal Chemistry; perspectives; future, challenges

\section{INTRODUÇÃO}

Os avanços da química medicinal têm origem com o desenvolvimento exponencial da química a partir do século XVIII. Naquela época, Joseph Priestley no Reino Unido, Carl Wilhelm Scheele na Suécia e Antoine Laurent de Lavoisier na França, fizeram contribuições pioneiras que revolucionaram o conhecimento da química, com destaque para os princípios da reatividade química, a teoria da combustão e o princípio da conservação da massa. ${ }^{1}$ No século seguinte, os trabalhos conduzidos por Joseph Louis Gay-Lussac e Justus Von Liebig estabeleceram os princípios químicos e físico-químicos que determinaram o crescimento dos recursos para as descobertas com finalidades terapêuticas. Poucas décadas depois, a química como disciplina científica encontrou uma aliada importante, quando os trabalhos de Claude Bernard, Rudolph Virchow e Louis Pasteur revolucionaram os conceitos em biologia e estabeleceram as bases e fundamentos para outras disciplinas como a fisiologia, bioquímica e microbiologia, que reunidas formaram o alicerce para o surgimento da farmacologia.

O século XIX também foi caracterizado pelo desenvolvimento de métodos de extração e purificação de materiais de origem natural que permitiram as primeiras descobertas dos princípios ativos em plantas (ex. morfina - isolada da cápsula de papoula em 1804; quinina - isolada da casca da chinchona em 1820; cocaína - isolada das folhas de coca em 1855) bem como o estabelecimento da química orgânica sintética que desenvolveu rotas para processos industriais que seriam aplicados na fabricação em larga escala dos primeiros medicamentos (ex. síntese do ácido acetilsalicílico, puro e estável, pelo químico Felix Hoffmann em 1897 e síntese em larga escala e comercialização da heroína pelo Laboratório Bayer em 1898). ${ }^{1,2}$

Os significativos avanços na química e biologia obtidos nos séculos XVIII e XIX proporcionaram a descoberta de inovações terapêuticas que marcaram o século XX. Durante esse período, centenas de novos fármacos foram desenvolvidos para doenças que até então não dispunham de tratamento. O século $\mathrm{XX}$ testemunhou o surgimento de novas classes terapêuticas como anti-inflamatórios (ex. corticoesteroides e anti-inflamatórios

*e-mail: rvcguido@ifsc.usp.br não-esteroidais); cardiotônicos (ex. digitálicos e nitroglicerina) e anti-hipertensivos (ex. diuréticos, bloqueadores do canal de $\mathrm{Ca}^{2+}$, inibidores $\alpha$ - e $\beta$-adrenérgicos, $\alpha-\beta$ bloquadores, inibidores da enzima conversora de angiotensina); antimicrobianos (ex. antibióticos, antifúngicos, antivirais); antitumorais (ex. alcaloides da vinca, taxol, antimetabólitos, inibidores de proteína quinase), antidiabéticos (ex. insulina, biguanidas, sulfonilureas, tiazolidinedionas, meglitinides); contraceptivos (ex. noretindrona, estradiol, progestina); antiúlcerosos (antagonistas de receptor $\mathrm{H}_{2}$, inibidores da bomba de $\mathrm{H}^{+}$); antilipêmicos (ex. estatinas); psicotrópicos (ex. antidepressivos, neurolépticos, ansiolíticos, anestésicos, antiepiléticos), entre outras. ${ }^{1,2}$

Além das inovações terapêuticas, o século XX foi marcado pela elucidação dos mecanismos biológicos em nível molecular. Em meados daquele século, os Laboratórios de Cavendish no King's College (Londres) reuniam um seleto grupo de pesquisadores, entre eles James Watson, Francis Crick, Maurice Wilkins, Rosalind E. Franklin e Linus Pauling, que tentavam determinar a estrutura do DNA. Os experimentos de cristalografia de raios X de Franklin e Wilkins forneceram informações valiosas sobre o DNA que foram reunidas, interpretadas e divulgadas no Modelo Watson-Crick da dupla hélice do DNA em 1953. ${ }^{3}$ A importância desse modelo para a história da ciência é inquestionável, pois proporcionou as bases moleculares das pesquisas em genética e bioquímica moderna. Com base nesses dados, Francis Crick postulou, em 1958, o "dogma central" da biologia molecular, ${ }^{4}$ que consiste na afirmação de que a informação genética é mantida e transferida em um processo unidirecional, passando de ácidos nucleicos para proteínas. Esse dogma norteou o surgimento da biotecnologia como uma nova área de pesquisa básica e aplicada. Desde então, houve uma verdadeira revolução biotecnológica com o surgimento de diversas novas disciplinas e procedimentos que visam à identificação funcional e/ou estrutural de tecidos, células, padrões de expressão gênicos e características metabólicas (ex. genômica, genômica funcional, proteômica, metabolômica e citômica). Os métodos e estratégias em biotecnologia têm fornecido informações extremamente úteis para a química biológica e química medicinal, incluindo o monitoramento de indicadores celulares ou bioquímicos associados às alterações fisiológicas e/ou metabólicas induzidas pelo estado de 
doença; e a avaliação dos efeitos dos fármacos sobre o organismo, proporcionando a descoberta de novos tratamentos para doenças. ${ }^{5}$

No século XXI, muitos serão os desafios que os químicos medicinais terão de enfrentar e superar para melhorar a qualidade de vida das populações. Os desafios consistem em doenças infecciosas e não-comunicáveis que estão surgindo e para compreendê-las e combatê-las tecnologias inovadoras deverão ser desenvolvidas. Nesse trabalho indicamos as tendências, perspectivas e oportunidades relacionadas à descoberta de fármacos bem como destacamos as ferramentas e estratégias que poderão ser utilizadas no desenvolvimento dos fármacos do século XXI.

\section{Quais serão os principais desafios para a Química Medicinal?}

\section{Doenças infecciosas - Novos antibióticos}

As infecções hospitalares causadas por bactérias Gram-negativas multirresistentes (MDR, do inglês, multidrug-resistant), extensivamente resistentes (XDR, do inglês, extensively drug-resistant) e panresistentes (PDR, do inglês, pan drug-resistant) aos fármacos disponíveis são uma grande ameaça mundial. ${ }^{6}$ Representantes destes patógenos incluem os micro-organismos denominados "ESKAPE", uma sigla utilizada partir das iniciais dos nomes das bactérias Enterococcus faecium, Staphylococcus aureus, Klebsiella pneumoniae, Acinetobacter baumannii, Pseudomonas aeruginosa $\mathrm{e}$ Enterobacter spp..$^{7}$ Esses micro-organismos desenvolveram mecanismos sofisticados de defesa para sobreviver à ameaça dos antibióticos, podendo-se citar: i. produção de enzimas inativadoras dos fármacos; ii. modificações dos antibióticos e/ou dos sítios alvo de ação dos mesmos; iii. superexpressão de sistemas de efluxo; iv. alterações da permeabilidade da membrana. Entre as estratégias genéticas de maior eficiência na determinação de resistência destaca-se a produção das carbapenemases, que são enzimas responsáveis pela hidrólise de fármacos $\beta$-lactâmicos (i.e., penicilinas, cefalosporinas, aztreonam, carbapenêmicos). Resistência aos carbapenêmicos em K. pneumoniae, $A$. baumannii e $P$. aeruginosa, principalmente determinada pela produção da enzima carbapenemase, surgiu em todo o mundo, com mortalidade superior a 50\%, fato atribuído principalmente à falta de regimes antimicrobianos eficazes. As opções de antibióticos para tratamento de infecções por bactérias produtoras de carbapenemases são muito limitadas e realizadas em associação de dois ou mais fármacos para que o tratamento tenha eficácia. ${ }^{8}$

Os avanços obtidos nas últimas décadas sobre mecanismos de ação, atividade in vitro, propriedades farmacocinéticas, farmacodinâmicas e toxicidade têm sido amplamente aplicados no desenvolvimento de novos candidatos a fármacos antibióticos, bem como na otimização de agentes antimicrobianos antigos, mas que apresentam excelente atividade contra micro-organismos do tipo ESKAPE. Exemplos destes fármacos capazes de superar os mecanismos de resistência atuais incluem colistina, tigeciclina, fosfomicina, temocillina e carbapenêmicos. Os estudos clínicos desses fármacos têm sido tratados em regime especial acelerado devido à grande necessidade e aumento da mortalidade causada por bactérias do tipo ESKAPE. Os mais novos fármacos aprovados ou em estudos avançados de Fase III para o combate de bactérias Gram-negativas da classe MDR e XDR incluem i. ceftolozano, cefalosporina de $5^{\mathrm{a}}$ geração administrada em associação com tazobactam, inibidor de $\beta$-lactamase (Zerbaxa $\left.{ }^{\circledR}\right)$; ii. avibactam, um inibidor sintético de $\beta$-lactamase não $\beta$-lactâmico, administrado em associação com ceftazidima (Avycaz ${ }^{\circledR}$ ); iii. plazomicina, neoglicosídeo derivado semi-sintético da sisomicina; iv. eravaciclina, derivado totalmente sintético da tetraciclina (Figura 1$).{ }^{8}$

\section{Doenças infecciosas - Novos antivirais}

Em geral, micro-organismos causadores de doenças infecciosas humanas são mantidos na natureza em ciclos que envolvem um vetor e um animal silvestre (ex. macaco ou pássaro). Entretanto, a modificação dos ecossistemas causada por razões econômicas, crescimento populacional desordenado, mudanças climáticas e aquecimento global tem contribuído substancialmente para o surgimento de doenças transmitidas por mosquitos vetores, em especial arboviroses como Chikungunya, Febre do Oeste do Nilo e Zika. ${ }^{9}$ A Chikungunya é causada por um vírus pertencente à família Togaviridae do gênero Alphavirus (CHIKV), enquanto a Febre do Oeste do Nilo (do inglês, West Nile Fever) e a Zika são causadas por vírus da família Flaviviridae do gênero Flavivirus (WNV e ZIKV, respectivamente) (Figura 2).

O impacto econômico dessas novas arboviroses é alarmante, pois alguns sintomas, como as fortes dores articulares da Chikungunya e a fadiga profunda da Febre do Oeste do Nilo, podem durar semanas ou meses, interferindo nas atividades produtivas do indivíduo. No caso da infecção por Zika, o paciente pode desenvolver a síndrome de Guillain-Barré, que causa fraqueza muscular generalizada e paralisia, além dos casos de microcefalia em bebês. ${ }^{10}$

Apesar de já conhecidas há bastante tempo, essas arboviroses não receberam grande atenção até a recente epidemia de Zika em 2015, que acendeu um sinal de alerta nos governos e organizações de saúde. Apesar dos intensos esforços, ainda não existem vacinas disponíveis, tampouco antivirais eficazes para o tratamento. ${ }^{14-17}$ Diante disso, a comunidade científica mundial tem se mobilizado intensamente para desenvolver alternativas que auxiliem na profilaxia e tratamento dessas infecções. Um exemplo marcante dessa mobilização foram os intensos e rápidos esforços internacionais para elucidar as proteínas constituintes do ZIKV. Em março de 2016 foi depositada a primeira estrutura de proteína de ZIKV no banco de dados de proteínas (PDB), ou seja, a estrutura do envelope viral determinada por Criomicroscopia Eletrônica (Cryo-EM). ${ }^{12}$ Exatamente um ano após, em março de 2017, já haviam 53 estruturas de proteínas do ZIKV, incluindo proteínas em complexo com ligantes e anticorpos (atualização de 29/03/2017). Esses dados estruturais, aliados às informações prévias sobre as enzimas homólogas do vírus da Dengue e seus respectivos inibidores candidatos a fármacos têm favorecido os avanços para a prevenção e tratamento das arboviroses. ${ }^{18}$

\section{Doenças infecciosas - Novos antiparasitários}

A prevalência das doenças tropicais negligenciadas (NTDs, do inglês, Neglected Tropical Diseases) é consequência marcante do subdesenvolvimento social e econômico dos países pobres. ${ }^{19-21}$ A Organização Mundial de Saúde (OMS) inclui neste grupo doenças infecciosas causadas por protozoários (ex. doença de Chagas, leishmaniose, doença do sono), vermes (ex. equinococose, helmintíases, oncocercíase, esquistossomose, cisticercose, dracunculíase, fasciolíase), bactérias (ex. lepra, tracoma, úlcera de Buruli e bouba) e vírus (ex. dengue, Chikungunya e raiva). Em maio de 2017, a $70^{a}$ Assembleia Mundial de Saúde, organizada pela OMS, aprovou uma resolução que incluiu mais doenças na classificação de NTD, sendo elas micetoma, micoses profundas e sarna. ${ }^{22}$

Essas doenças tropicais afetam milhões de indivíduos em todo o mundo, entre homens, mulheres e crianças. Para a maioria destas doenças, que causam altos índices de morbidade e mortalidade, os tratamentos disponíveis são limitados e ineficazes, e o quadro é constantemente agravado pela emergência de cepas resistentes. Estima-se que do total investido em Pesquisa, Desenvolvimento e Inovação (PD\&I) de fármacos, somente $10 \%$ são destinados a doenças que representam cerca de $90 \%$ dos problemas globais de saúde humana. ${ }^{23}$ Visando reverter esse panorama, iniciativas envolvendo parcerias efetivas na tríade governo-universidade-empresa vêm sendo estabelecidas. ${ }^{20}$ A Medicines for Malaria Venture (MMV) $)^{24}$ e a 


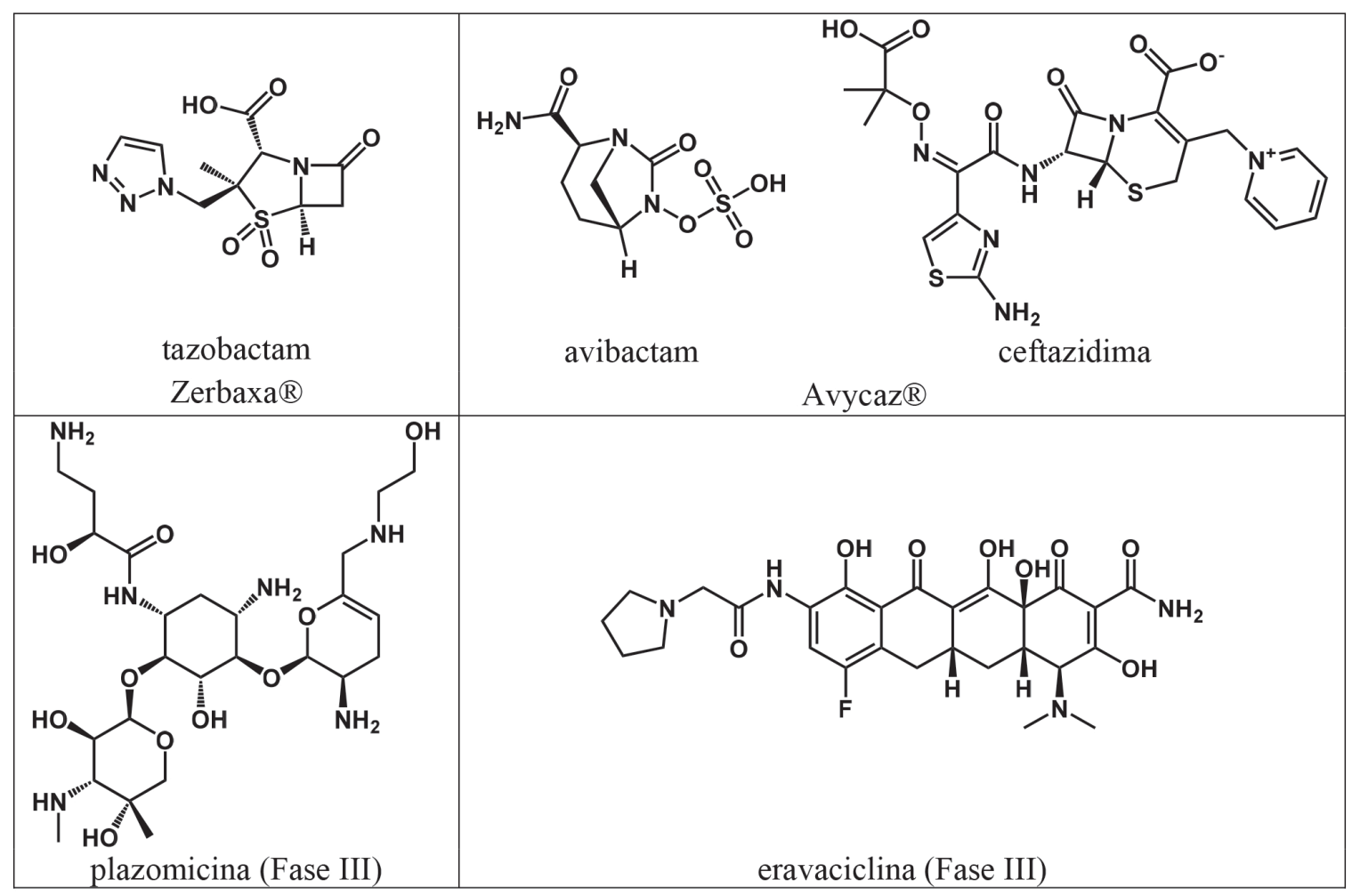

Figura 1. Estruturas químicas de novos fármacos aprovados e candidatos a fármacos em estudos avançados de Fase III para o combate de bactérias Gram-negativas da classe multirresistentes (MDR) e extensivamente resistentes (XDR)
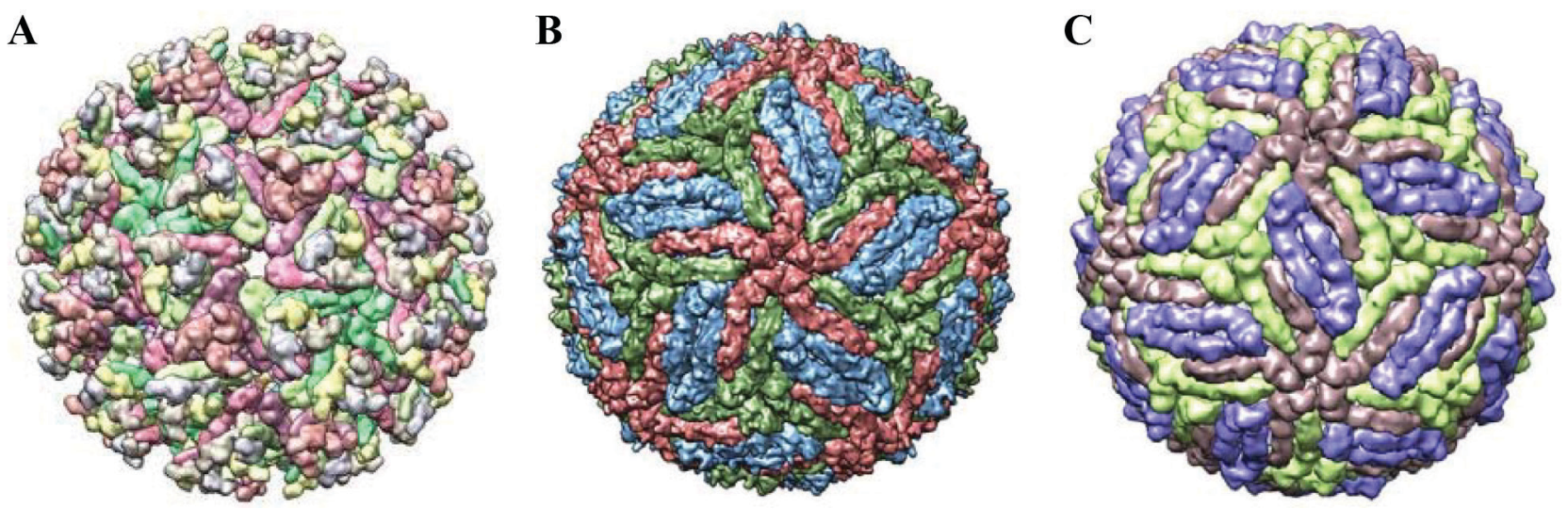

Figura 2. Estrutura $3 D$ do envelope dos vírus (A) Chikungunya $(P D B I D, 3 J 2 W),{ }^{11}(\boldsymbol{B})$ Zika $(P D B I D, 5 I R E)^{12}$ e (C) Febre do Oeste do Nilo (PDB ID, 3JOB $)^{13}$

Drugs for Neglected Diseases initiative (DNDi) ${ }^{25}$ são exemplos de parcerias público-privadas que tem como objetivo criar, estimular e investir em PD\&I de fármacos contra uma variedade de doenças tropicais. A MMV e DNDi juntas tem em seu portfólio de produtos uma lista de 10 medicamentos desenvolvidos nos últimos 10 anos para o tratamento de doenças parasitárias (Tabela 1).

Os avanços obtidos através dessas parcerias são sensíveis e de alto impacto para as sociedades mundiais. Por exemplo, o relatório mundial de malária de 2016 destaca progressos significativos como: i. cerca de 212 milhões de casos de malária ocorreram em todo o mundo, indicando uma queda de $22 \%$ desde 2000 e de $14 \%$ desde 2010; ii. estima-se que 429.000 mortes por malária ocorreram globalmente, ou seja, uma diminuição de $50 \%$ desde 2000 e de $22 \%$ desde 2010; iii. 303.000 mortes por malária ocorreram em crianças com menos de 5 anos, um número que, apesar de alarmante, representa uma diminuição de 60\% desde 2000 e em $29 \%$ desde 2010; iv. dos 91 países e territórios com transmissão de malária em 2015, estima-se que 40 tenham alcançado uma redução nas taxas de incidência de $40 \%$ ou mais entre 2010 e 2015 ; e v. estima-se que as taxas de mortalidade por malária tenham diminuído em $62 \%$ globalmente entre 2000 e 2015 , e em $29 \%$ entre 2010 e 2015 . $^{26}$

A meta \#3 da agenda 2030 para o desenvolvimento sustentável, publicada pela Organização das Nações Unidas, estabelece: "Apoiar a investigação e o desenvolvimento de vacinas e medicamentos para as doenças transmissíveis e não transmissíveis que afetam principalmente os países em desenvolvimento e proporcionar acesso a medicamentos e vacinas essenciais. ${ }^{27}$ Portanto, avanços significativos serão obtidos para as doenças tropicais, incluindo as atualmente denominadas doenças negligenciadas. Diante desse cenário, a OMS tem como perspectiva para a próxima década a eliminação da leishmaniose visceral em países anteriormente com alta taxa de incidência e a erradicação da dracunculíase. ${ }^{28,29}$ Além disso, DNDi tem como objetivo o lançamento de 16 a 18 novos tratamentos para as doenças parasitárias até 2023 e MMV tem atualmente em seu portfólio de projetos 16 candidatos a fármacos em estudos pré-clínicos e clínicos..$^{24,25}$ 
Tabela 1. Medicamentos para doenças parasitárias desenvolvidos pela MMV e DNDi nos últimos 10 anos

\begin{tabular}{|c|c|c|c|c|c|}
\hline Medicamento & Fármaco & Indicação & Desenvolvedor & Ano de lançamento & Parceiro \\
\hline $\begin{array}{c}\text { ASAQ } \\
\text { Winthrop }\end{array}$ & artesunato + amodiaquina & Malária & MMV / DNDi & 2007 & Sanofi \\
\hline ASMQ & artesunato + mefloquina & Malária & MMV / DNDi & 2008 & $\begin{array}{l}\text { Farmanguinhos/ } \\
\text { Fiocruz }\end{array}$ \\
\hline Artesun & artesunato (injetável) & Malária & MMV & 2011 & $\begin{array}{c}\text { Guillin } \\
\text { Pharmaceuticals }\end{array}$ \\
\hline $\begin{array}{c}\text { Coartem } \\
\text { Dispersible }\end{array}$ & arteméter-lumefantrina & Malária & MMV & 2009 & Novartis \\
\hline Eurartesim & $\begin{array}{c}\text { diidroartemisinina }+ \\
\text { piperaquina }\end{array}$ & Malária & MMV & 2011 & Sigma-Tau \\
\hline Pyramax & pironaridina + artesunato & Malária & MMV & 2012 & $\begin{array}{c}\text { Shin Poong } \\
\text { Pharmaceutical }\end{array}$ \\
\hline SPAQ & $\begin{array}{l}\text { sulfadoxina }+ \text { pirimetami- } \\
\text { na }+ \text { amodiaquina }\end{array}$ & Malária & MMV & 2012 & Guillin Pharmaceuticals \\
\hline NECT & $\begin{array}{c}\text { eflornitina (IV) e nifurti- } \\
\text { mox (oral) }\end{array}$ & Doença do Sono & DNDi & 2009 & Sanofi / Bayer \\
\hline Benznidazol pediatrico & benznidazol & Doença de Chagas & DNDi & 2011 & LAFEPE / ELEA \\
\hline SSG\&PM & $\begin{array}{l}\text { estibogluconato de sódio } \\
+ \text { paromomicina }\end{array}$ & Leishmaniose Visceral & DNDi & 2010 & LEAP / MSF / WHO \\
\hline
\end{tabular}

\section{Doenças não-comunicáveis - Desordens neurodegenerativas}

$\mathrm{O}$ envelhecimento populacional é caracterizado pelo constante aumento da expectativa de vida e a redução da fecundidade. Esse fenômeno ocorre em escala global sendo mais observado nos países desenvolvidos. Dados da Organização das Nações Unidas (ONU) indicam que em 1950 existiam 250 milhões de indivíduos com mais de 60 anos no planeta. Esse número praticamente quadruplicou até o ano 2015, somando 901 milhões de pessoas (12\% da população mundial que contabilizava 7,3 bilhões de pessoas em 2015). As projeções para 2030 indicam que o número de pessoas no mundo com 60 anos ou mais deverá crescer para 1,4 bilhão, e até 2050 a população global de idosos será de 2,1 bilhões, ou seja, $22 \%$ da população mundial terá mais de 60 anos (a projeção da população mundial é de 9,7 bilhões de pessoas em 2050). ${ }^{30}$ Nesse cenário, as doenças relacionadas ao envelhecimento terão impacto ainda maior, entre elas, destacam-se as doenças neurodegenerativas como a doença de Alzheimer e a doença de Parkinson. Para se ter uma ideia do impacto destas doenças no futuro, a OMS divulgou em 2012 um relatório sobre a doença de Alzheimer e as demências associadas ao envelhecimento, no qual solicita aos governantes para tornarem estas doenças prioridade em políticas de saúde pública. ${ }^{31}$ Já a doença de Parkinson, uma doença neurodegenerativa crônica e progressiva, que apesar de em 2017 ter completado 200 anos da sua primeira descrição por James Parkinson, ${ }^{32}$ permanece até hoje com várias questões a serem respondidas, incluindo a sua causa, melhor forma de diagnóstico precoce e prevenção.

Atualmente, as alternativas terapêuticas para ambas as doenças são bastante limitadas. Embora eficazes em alguns casos, os medicamentos disponíveis atuam de forma paliativa, portanto, novos fármacos serão extremamente necessários. Para tanto, intensos estudos de pesquisa básica têm buscado elucidar as bases moleculares envolvidas na patologia dessas doenças. ${ }^{33}$ Paralelamente, esses trabalhos têm indicado novos alvos moleculares para a descoberta de fármacos. Por exemplo, a doença de Parkinson é patologicamente caracterizada pela presença de corpos de Lewy e neurites de Lewy, que consistem em agregados intracitoplasmáticos ricos em fibrilas de $\alpha$-sinucleína. ${ }^{34}$ Esta mesma fibrila está associada à regulação da fibrilização das proteínas $\beta$-amiloide $(\mathrm{A} \beta)$ e tau, ambas proteínas-chave na fisiopatologia da doença de Alzheimer. ${ }^{35,36}$ A estrutura da $\alpha$-sinucleína foi determinada por espectroscopia de RMN de estado sólido e validada por microscopia eletrônica e difração de raios X ${ }^{37} \mathrm{~A}$ estrutura em alta resolução corresponde a uma forma que induz a patologia e, portanto, auxilia na compreensão das bases moleculares para o recrutamento e agregação de $\alpha$-sinucleína bem como para o desenvolvimento de candidatos a fármacos para o tratamento da doença de Parkinson (Figura 3A). Recentemente, foi demonstrado que a doxiciclina, um antibiótico pertencente à família das tetraciclinas (Figura 3B), possui atividade neuroprotetora em modelos animais. $\mathrm{O}$ mecanismo de ação proposto seria através da interação da doxiciclina com os intermediários de agregação da $\alpha$-sinucleína, impedindo, portanto, a formação de fibrilas de $\alpha$-sinucleína. ${ }^{38}$

\section{Doenças não-comunicáveis - Doenças autoimunes}

As doenças autoimunes são desordens imunológicas caracterizadas pela diminuição da tolerância aos componentes do próprio organismo. As causas das doenças autoimunes ainda não são claras, contudo, há indícios que elas estejam relacionadas às alterações no processo de diferenciação de antígenos externos e os do próprio organismo de um indivíduo, predisposição genética, alterações nos níveis hormonais e baixo controle imuno-regulatório. ${ }^{39}$ Há pelo menos 80 doenças autoimunes, entre elas citam-se: artrite reumatoide, psoríase, lúpus eritematoso sistêmico, doença celíaca, vasculite, diabetes tipo 1 e esclerose múltipla.

Até o final do século passado, os indivíduos acometidos por essas condições contavam com medicamentos que apenas aliviavam os sintomas das doenças autoimune. Contudo, os avanços na produção de proteínas, na cultura de células e hibridomas permitiram o desenvolvimento de uma nova classe terapêutica denominada de "biofármacos" ou "proteínas terapêuticas" ${ }^{40}$ Os pacientes portadores de artrite reumatoide e psoríase foram os primeiros a ter acesso a um tratamento eficaz para doenças autoimunes. O desenvolvimento de anticorpos monoclonais não apenas ofereceu uma alternativa eficiente de tratamento, mas também abriu as portas para um mercado 

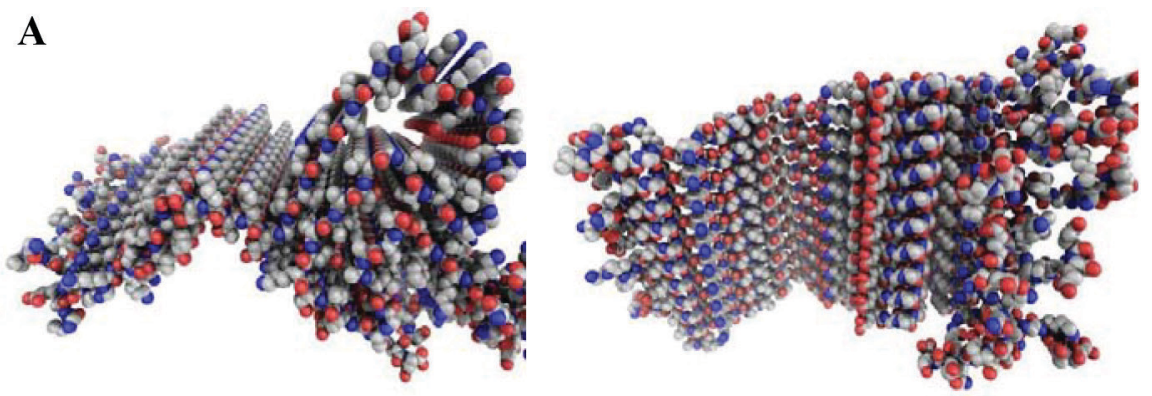

B<smiles>C[C@H]1[C@@H](C)c2cccc(O)c2C(=O)C2=C(O)[C@]3(O)C(=O)C(C(N)=O)=C(O)[C@H](N(C)C)[C@]3(C)[C@H]21</smiles>

Figura 3. (A) Visão ortogonal da estrutura da $\alpha$-sinucleína obtida por RMN de estado sólido (PDB ID, 2NOA). (B) Estrutura molecular da doxiciclina

extremamente promissor. Atualmente, entre os 10 maiores sucessos de vendas de medicamentos para doenças autoimunes, 8 são proteínas terapêuticas (Tabela 2). As perspectivas apontam que em breve, portadores de lúpus, doença celíaca e esclerose múltipla também terão possibilidade de tratamento através do desenvolvimento de novas proteínas terapêuticas.

\section{Quais serão as novas tecnologias em Química Medicinal?}

O estudo das relações entre a estrutura e atividade (do inglês, SAR, structure-activity relationships) continuará a ser o pilar da Química Medicinal. Nesse sentido, ensaios para avaliação de atividade biológica e propriedades toxicológicas serão cada vez mais sofisticados e incluirão organoides (i.e., mini órgão tridimensional cultivado in vitro que apresenta microanatomia realista) e métodos relacionados que diminuirão a necessidade de estudos em animais. Além disso, estes métodos fornecerão informações mais relevantes e precisas, uma vez que, por serem constituídos por células humanas, os organoides oferecem uma oportunidade para criar modelos celulares de doenças humanas, que podem ser estudados em laboratório para melhor compreensão das causas da doença e identificação de possíveis tratamentos. ${ }^{41}$ Paralelamente, novos métodos sintéticos serão desenvolvidos, e serão baseados em rotas e processos que reduzem ou eliminam o uso e geração de substâncias nocivas ao meio ambiente, através de i. sistemas de solventes menos tóxicos (ou até mesmo a ausência deles), ii. processos catalíticos e rotas sintéticas mais eficientes, iii. técnicas de análise e isolamento mais robustas. ${ }^{42}$

A avaliação da segurança e eficácia de compostos químicos se tornou uma etapa essencial na regulação de fármacos, cosméticos, agroquímicos, entre outros. Historicamente, esta avaliação tem sido feita através do uso de animais e, consequentemente, é notório que a sociedade tenha tolerado cada vez menos o uso de animais em laboratório. ${ }^{43,44}$ Nesse sentido, os métodos alternativos ao uso de animais tem ganhado cada vez mais espaço. Como exemplo, a experimentação animal para produtos cosméticos já é proibida na União Europeia desde $2003,{ }^{45}$ sendo que em 2009 proibiu-se a venda de produtos cosméticos assim testados. No Brasil, a Agência Nacional de Vigilância Sanitária (ANVISA) aprovou em 2015 uma resolução que permite que métodos alternativos ao uso de animais, ${ }^{46}$ já reconhecidos no país pelo Conselho Nacional de Controle de Experimentação Animal (CONCEA), sejam suficientes para pedidos de registro de medicamentos, cosméticos, produtos para saúde, produtos de limpeza, entre outros produtos. A partir de 2019, a experimentação animal não será mais permitida para produtos que tenham métodos alternativos reconhecidos pelo CONCEA, como exemplo irritação ocular, sensibilização da pele, toxicidade aguda, entre outros.

A análise rápida e precisa de grandes quantidades de dados será de fundamental importância para a descoberta de novos fármacos, como também para o diagnóstico e seleção de tratamento personalizado. Nesse contexto, quimioinformática, uma área interdisciplinar

Tabela 2. Os 10 medicamentos com maior volume de vendas para doenças autoimunes (vendas nos EUA)

\begin{tabular}{|c|c|c|c|c|c|c|}
\hline Posição & Medicamento & Princípio Ativo & Indicação & Categoria & Fabricante & Vendas*(US\$, bilhões) \\
\hline 1 & Humira & adalimumabe & $\begin{array}{l}\text { artrite reumatoide / doença } \\
\text { de Crohn / psoríase }\end{array}$ & Anticorpo Monoclonal & Abbvie & 10,65 \\
\hline 2 & Enbrel & etanercepte & $\begin{array}{l}\text { artrite reumatoide / } \\
\text { artrite psoriática }\end{array}$ & Proteína Fusionada & Amgen & 6,62 \\
\hline 3 & Remicade & infliximabe & $\begin{array}{l}\text { artrite reumatoide / doença } \\
\text { de Crohn / artrite psoriática }\end{array}$ & Anticorpo Monoclonal & Janssen & 4,98 \\
\hline 4 & Stelara & ustequinumabe & psoríase & Anticorpo Monoclonal & Janssen & 2,04 \\
\hline 5 & Orencia & abatacepte & artrite reumatoide & Proteína Fusionada & Bristol- Myers Squibb & 1,32 \\
\hline 6 & Cimzia & certolizumabe pegol & $\begin{array}{l}\text { artrite reumatoide / doença } \\
\text { de Crohn / psoríase }\end{array}$ & Fragmento Fab & $\mathrm{UCB}$ & 1,02 \\
\hline 7 & Simponi & golimumabe & $\begin{array}{l}\text { artrite reumatoide / } \\
\text { artrite psoriática }\end{array}$ & Anticorpo Monoclonal & Janssen & 0,65 \\
\hline 8 & Actemra & tocilizumabe & $\begin{array}{c}\text { artrite reumatoide / } \\
\text { artrite idiopática juvenil }\end{array}$ & Anticorpo Monoclonal & Genentech & 0,55 \\
\hline 9 & Xeljanz & tofacitinibe & artrite reumatoide & Inibidor de Janus kinase & Pfizer & 0,54 \\
\hline 10 & Otezla & apremilast & $\begin{array}{c}\text { psoríase / artrite } \\
\text { psoriática }\end{array}$ & Inibidor de PDE4 & Celgene & 0,51 \\
\hline de ven & medicament & para doenças aut & & & & 30,2 \\
\hline
\end{tabular}

*Fonte: IMS Health (ano base 2015) 
que utiliza recursos computacionais e de tecnologia da informação para transformar informação química em conhecimento, ${ }^{47,48}$ tem se consolidado e sua utilidade e impacto na química medicinal tende a aumentar nas próximas décadas. Isto se justifica porque bases de dados com informações químicas relevantes e propriedades biológicas têm expandido rapidamente e sua exploração e transformação da informação gerada em conhecimento é essencial para a descoberta de novos compostos com atividade ou propriedades desejadas. ${ }^{48}$

Com relação ao tratamento personalizado, este será integrado à informação pessoal de cada indivíduo, que carregará um chip com seu próprio genoma e proteoma, permitindo assim a escolha dos melhores medicamentos e adaptação do tratamento às características moleculares do indivíduo. ${ }^{49}$ Essas informações poderão ser utilizadas em ensaios clínicos, no quais o conhecimento sobre o genoma/ proteoma de cada indivíduo irá revolucionar a análise dos resultados, substituindo a abordagem simplista de médias globais por uma análise estatística superior e que fornecerá subsídios para conclusões muito mais adequadas. Nesse contexto, os avanços na tecnologia microfluídica terão função central. ${ }^{50,51}$ Atualmente, procedimentos de biologia molecular e análise de DNA já empregam chips com tecnologia microfluídica para a detecção, diganóstico, pré-tratamento e preparação de amostras. ${ }^{52-55}$

A determinação da estrutura de macromoléculas e de grandes complexos biomacromoleculares por criomicroscopia eletrônica (Cryo-EM) tornar-se-ão importantes para o planejamento de novos fármacos. Atualmente, diversos paradigmas sobre a Cryo-EM já foram quebrados, por exemplo, i. determinação em resolução próxima a atômica $(3,8 \AA)$ de estrutura de enzima com massa molecular menor que $100 \mathrm{kDa}$; ii. determinação de complexos proteína-inibidor e proteína-proteína; iii. identificação de mudanças conformacionais induzidas pela interação com ligantes. ${ }^{56}$ Esses dados têm indicado o potencial desse método para as mais diversas aplicações em Biologia Estrutural e Química Medicinal. ${ }^{57}$

A disponibilidade de informação estrutural qualificada e de alta resolução sobre canais, receptores e transportadores em membranas favorecerão a melhor compreensão dos fatores que determinam a biodisponibilidade e estabilidade metabólica de compostos candidatos a fármacos. Além disso, melhores ferramentas computacionais serão desenvolvidas na área de propriedades farmacocinéticas de absorção, distribuição, metabolismo e excreção (ADME) assim como de toxicidade, de modo a tornar os estudos em Química Medicinal mais eficientes. ${ }^{58}$

Em meados do século XXI, os métodos e o poder computacional terão evoluído significativamente para tornar possível predizer e classificar de forma bastante acurada a afinidade de ligantes por seus alvos moleculares. A capacidade de investigar longos tempos de simulação oferecerá oportunidades de cálculos de afinidade com maior precisão (ex. erro $<1 \mathrm{kcal} \mathrm{mol}^{-1}$ ). Essa possibilidade é particularmente interessante quando se planeja uma molécula altamente atrativa do ponto de vista computacional, mas que pode ser sinteticamente desafiadora. Nesse sentido, a aplicação de métodos como o de perturbação da energia livre (FEP, do inglês, Free Energy Perturbation) em um projeto de descoberta de fármacos será de fundamental importância para reduzir o número de compostos com atividade inadequada bem como otimizar o tempo de descoberta de reais candidatos a fármacos. ${ }^{59}$

\section{CONSIDERAÇÕES FINAIS}

A humanidade testemunhou nos últimos três séculos avanços extremamente importantes nas áreas de Química e Biologia que revolucionaram o modo e qualidade de vida das diversas populações. Entre os avanços que se mostraram essenciais para a descoberta e desenvolvimento de novos fármacos destacam-se: i. a obtenção dos princípios ativos extraídos de produtos naturais, ii. o estabelecimento de rotas sintéticas de alta eficiência, iii. a melhor compreensão dos processos fisiopatológicos, iv. o estabelecimento de métodos biotecnológicos, v. a elucidação das estruturas 3D de pequenas e macromoléculas, e vi. o desenvolvimento de métodos computacionais que auxiliam a predição de propriedades farmacodinâmicas e farmacocinéticas. Todas essas estratégias e tecnologias impactaram de modo positivo e significativo o processo de descoberta e desenvolvimento de novos fármacos, os quais evoluíram de uma abordagem do tipo "tentativa e erro", para uma abordagem racional focada nos mecanismos fisiopatológicos envolvidos, no conhecimento do alvo molecular de ação e no equilíbrio entre a eficácia e segurança de novas moléculas candidatas a fármacos.

Estamos vivendo nesse início de século XXI a chamada "Quarta Revolução Industrial”. ${ }^{60}$ A Primeira Revolução Industrial se estabeleceu com o uso de maquinário à base de água e vapor, para produção em larga escala; a Segunda correspondeu ao uso da energia elétrica; já a Terceira, surgiu a partir da aplicação de eletrônica e tecnologias de informação. A Quarta Revolução Industrial, também conhecida como "Indústria 4.0", será marcada pela convergência de tecnologias digitais, físicas e biológicas e caracterizada pelos processos produtivos que utilizarão robôs integrados em sistemas ciberfísicos. Temas como nanotecnologias, neurotecnologias, robôs, inteligência artificial, biotecnologia, sistemas de armazenamento de energia, drones e impressoras 3D farão cada vez mais parte do cotidiano da humanidade e terão impacto profundo no modo como fazemos ciência.

Do ponto de vista da Química Medicinal brasileira, evoluímos significativamente nas últimas décadas, ${ }^{61}$ mas para que possamos superar os desafios e aproveitar ao máximo as oportunidades do século XXI, devemos nos posicionar no cenário mundial como um país produtor de inovações farmacêuticas. Para tanto, precisamos cada vez internacionalizar a pesquisa através do estabelecimento de colaborações nas quais os objetivos sejam comuns para todos os membros da equipe e cada parceiro desempenhe, com comprometimento e eficiência, a sua função específica. Nosso País necessita de exemplos de sucesso na área estratégica de fármacos e medicamentos para motivar a vinda de novos parceiros científicos e comerciais e assim mostrar o real potencial da ciência brasileira. Portanto, como desafio específico para a ciência brasileira na área de Química Medicinal e Química Biológica, precisamos investir fortemente em pesquisas translacionais, ou seja, incentivar cada vez mais o seu início na ciência básica que fornecerá dados sólidos e consistentes para subsidiar a ciência aplicada que levará ao desenvolvimento de inovações terapêuticas de elevado impacto social e econômico, caracterizando assim a conclusão dos estudos com aplicação prática do conhecimento desenvolvido.

\section{AGRADECIMENTOS}

Os autores agradecem à FAPESP (Fundação de Amparo à Pesquisa do Estado de São Paulo), CNPq (Conselho Nacional de Desenvolvimento Científico e Tecnológico), CAPES (Coordenação de Aperfeiçoamento de Pessoal de Nível Superior), FAPERJ (Fundação de Amparo à Pesquisa do Estado do Rio de Janeiro) e FAPEG (Fundação de Amparo à Pesquisa do Estado de Goiás) pelo apoio a pesquisa na área de descoberta e desenvolvimento de fármacos. Ao Prof. Eliezer J. Barreiro pelo convite para apresentar a palestra intitulada "Medicinal Chemistry at 21st Century: Perspectives from Division of Medicinal Chemistry" durante a sessão temática do BrazMedChem 2016, evento que motivou a redação desse trabalho. Aos Profs. Wim G. J. Hol (University of Washington), Paul A. Michels (University of Edinburgh) Glaucius Oliva (Universidade de São Paulo) e Maria Cristina Nonato Costa (Universidade de São Paulo) pelas sugestões e discussões científicas que foram essenciais para 
a elaboração do texto. À Divisão de Química Medicinal e Química Biológica e à Sociedade Brasileira de Química por oferecer oportunidades de debatermos temas relevantes para o desenvolvimento da ciência brasileira.

\section{REFERÊNCIAS}

1. Wermuth, C. G.; The practice of medicinal chemistry; Elsevier/ Academic Press, 2008.

2. Foye, W. O.; Lemke, T. L.; Williams, D. A.; Foye's principles of medicinal chemistry, Lippincott Williams \& Wilkins, 2008.

3. Watson, J. D.; Crick, F. H. C.; Nature 1953, 171, 737.

4. Crick F. H. C.; Nature 1970, 227, 561.

5. Guido, R. V. C.; Andricopulo, A. D.; Oliva, G.; Estudos Avançados 2010, $24,81$.

6. Poulakou, G.; Bassetti, M.; Righi, E.; Dimopoulos, G.; Future Microbiol. 2014, 9, 1053.

7. Boucher, H. W.; Talbot, G. H.; Bradley, J. S.; Edwards, J. E.; Gilbert, D.; Rice, L. B.; Scheld, M.; Spellberg, B.; Bartlett, J.; Clin. Infect. Dis. 2009, 48, 1.

8. Karaiskos, I.; Giamarellou, H.; Expert Opin. Pharmacother. 2014, 15, 1.

9. Lima-Camara, T. N.; Rev. Saúde Pública 2016, 50, 1.

10. Faria, N. R.; Azevedo, R. do S. da S.; Kraemer, M. U. G.; Souza, R.; Cunha, M. S.; Hill, S. C.; Thézé, J.; Bonsall, M. B.; Bowden, T. A.; Rissanen, I.; Rocco, I. M.; Nogueira, J. S.; Maeda, A. Y.; Vasami, F. G. da S.; Macedo, F. L. de L.; Suzuki, A.; Rodrigues, S. G.; Cruz, A. C. R.; Nunes, B. T.; Medeiros, D. B. de A.; Rodrigues, D. S. G.; Queiroz, A. L. N.; da Silva, E. V. P.; Henriques, D. F.; da Rosa, E. S. T.; de Oliveira, C. S.; Martins, L. C.; Vasconcelos, H. B.; Casseb, L. M. N.; Simith, D. de B.; Messina, J. P.; Abade, L.; Lourenço, J.; Alcantara, L. C. J.; de Lima, M. M.; Giovanetti, M.; Hay, S. I.; de Oliveira, R. S.; Lemos, P. da S.; de Oliveira, L. F.; de Lima, C. P. S.; da Silva, S. P.; de Vasconcelos, J. M.; Franco, L.; Cardoso, J. F.; Vianez-Júnior, J. L. da S. G.; Mir, D.; Bello, G.; Delatorre, E.; Khan, K.; Creatore, M.; Coelho, G. E.; de Oliveira, W. K.; Tesh, R.; Pybus, O. G.; Nunes, M. R. T.; Vasconcelos, P. F. C.; Science 2016, 352, 345.

11. Sun, S.; Xiang, Y.; Akahata, W.; Holdaway, H.; Pal, P.; Zhang, X.; Diamond, M. S.; Nabel, G. J.; Rossmann, M. G.; eLife 2013, 2 , e00435.

12. Sirohi, D.; Chen, Z.; Sun, L.; Klose, T.; Pierson, T. C.; Rossmann, M. G.; Kuhn, R. J.; Science, 2016, 352, 467.

13. Zhang, W.; Kaufmann, B.; Chipman, P. R.; Kuhn, R. J.; Rossmann, M. G.; J. Struct. Biol. 2013, 183, 86.

14. Cheng, F.; Murray, J. L.; Rubin, D. H.; Trends Mol. Med. 2016, $22,919$.

15. Ekins, S.; Mietchen, D.; Coffee, M.; Stratton, T. P.; Freundlich, J. S.; Freitas-Junior, L.; Muratov, E.; Siqueira-Neto, J.; Williams, A. J.; Andrade, C.; F1000Research 2016, 5, 150.

16. Ekins, S.; Perryman, A. L.; Horta Andrade, C.; PLoS Negl. Trop. Dis. 2016, 10 .

17. Fernandez, E.; Diamond, M. S.; Curr. Opin. Virol. 2017, 23, 59-67.

18. Godoy, A. S.; Lima, G. M. A.; Oliveira, K. I. Z.; Torres, N. U.; Maluf, F. V; Guido, R. V. C.; Oliva, G.; Nat. Commun. 2017, 8, 14764.

19. Laursen, L.; Nature 2016, 533, S68.

20. Dias, L. C.; Dessoy, M. A.; Guido, R. V. C.; Oliva, G.; Andricopulo, A. D.; Quim. Nova 2013, 36, 1552.

21. Field, M. C.; Horn, D.; Fairlamb, A. H.; Ferguson, M. A. J.; Gray, D. W.; Read, K. D.; De Rycker, M.; Torrie, L. S.; Wyatt, P. G.; Wyllie, S.; Gilbert, I. H.; Nat. Rev. Microbiol. 2017, 15, 217.

22. WHO; http://www.who.int/neglected_diseases/diseases/en/, acessada em dezembro de 2017.

23. WHO; World health statistics, 2015.

24. MMV - Medicine for Malaria Venture; http:/www./mmv.org, acessada em dezembro de 2017.
25. DNDi; https://www.dndi.org/, acessada em dezembro de 2017.

26. WHO; World Malaria Report, 2016.

27. United Nations General Assembly; https://sustainabledevelopment. un.org/content/documents $/ 21252030 \% 20$ A genda $\% 20$ for $\% 20$ Sustainable\%20Development\%20web.pdf, 2015, 1.

28. Visceral leishmaniasis: WHO publishes validation document as countries approach elimination http://www.who.int/neglected_diseases/ news/Visceral_leishmaniasis_WHO_publishes_validation_document/ en/, acessada em dezembro de 2017.

29. Dracunculiasis eradication: Mali reports zero cases in 2016; http:// www.who.int/neglected_diseases/news/Mali_reports_zero_cases_ in_2016/en/, acessada em dezembro de 2017.

30. ONU; Suggest. Cit. United Nations, Dep. Econ. Soc. Aff. Popul. Div. (2015). World Popul. Ageing 2015, United Nations, 164.

31. Alzheimer's Disease International; http://www.alz.co.uk/WHOdementia-report, acessada em dezembro de 2017.

32. Hurwitz, B.; Lancet 2017, 389, 2098.

33. Riek, R.; Eisenberg, D. S.; Nature 2016, 539, 227.

34. Goedert, M.; Jakes, R.; Spillantini, M. G.; J. Parkinsons. Dis. 2017, 7, S51.

35. Fitzpatrick, A. W. P.; Falcon, B.; He, S.; Murzin, A. G.; Murshudov, G.; Garringer, H. J.; Crowther, R. A.; Ghetti, B.; Goedert, M.; Scheres, S. H. W.; Nature 2017, 547, 185.

36. Wong, Y. C.; Krainc, D.; Nat. Med. 2017, 23, 1.

37. Tuttle, M. D.; Comellas, G.; Nieuwkoop, A. J.; Covell, D. J.; Berthold, D. A.; Kloepper, K. D.; Courtney, J. M.; Kim, J. K.; Barclay, A. M.; Kendall, A.; Wan, W.; Stubbs, G.; Schwieters, C. D.; Lee, V. M. Y.; George, J. M.; Rienstra, C. M.; Nat. Struct. Mol. Biol. 2016, 23,1 .

38. González-Lizárraga, F.; Socías, S. B.; Ávila, C. L.; Torres-Bugeau, C. M.; Barbosa, L. R. S.; Binolfi, A.; Sepúlveda-Díaz, J. E.; Del-Bel, E.; Fernandez, C. O.; Papy-Garcia, D.; Itri, R.; Raisman-Vozari, R.; Chehín, R. N.; Sci. Rep. 2017, 7, 41755.

39. Rioux, J. D.; Abbas, A. K.; Nature 2005, 435, 584.

40. Leader, B.; Baca, Q. J.; Golan, D. E.; Nat. Rev. Drug Discov. 2008, 7 , 21.

41. Lancaster, M. A.; Renner, M.; Martin, C.-A.; Wenzel, D.; Bicknell, L. S.; Hurles, M. E.; Homfray, T.; Penninger, J. M.; Jackson, A. P.; Knoblich, J. A.; Nature 2013, 501, 373.

42. Zhang, J.; Cue, B. W.; Green Techniques for Organic Synthesis and Medicinal Chemistry, John Wiley \& Sons, Ltd: Chichester, 2012; pp. 631-658.

43. Jẹdrzejewska, S.; ALTEX 2014, 31, 371.

44. Aziz, T.; Stein, J.; Yogeshwar, R.; Nature 2011, 470, 457.

45. European Union; Journal officiel de l'Union européenne, 2003, 26.

46. Anvisa; Resolução No 35 DE 07 de agosto de 2015, 1.

47. Gasteiger, J.; Engel, T.; Chemoinformatics: A Textbook, Wiley: Hoboken, 2003

48. Alves, V.; Braga, R.; Muratov, E.; Andrade, C.; Quim. Nova 2017, X, 1. 49. Katsnelson, A.; Nature 2010.

50. deMello, A. J.; Nature 2006, 442, 394.

51. Wootton, R. C. R.; deMello, A. J.; Nature 2012, 483, 43

52. deMello, A. J.; Nature 2003, 422, 28.

53. Oliveira, K. A.; de Oliveira, C. R.; da Silveira, L. A.; Coltro, W. K. T.; Analyst 2013, 138, 1114.

54. De Souza, F. R.; Alves, G. L.; Coltro, W. K. T.; Anal. Chem. 2012, 84, 9002.

55. Coltro, W. K. T.; Cheng, C. M.; Carrilho, E.; de Jesus, D. P.; Electrophoresis 2014, 35, 2309.

56. Merk, A.; Bartesaghi, A.; Banerjee, S.; Falconieri, V.; Rao, P.; Davis, M. I.; Pragani, R.; Boxer, M. B.; Earl, L. A.; Milne, J. L. S.; Subramaniam, S.; Cell 2016, 165, 1698

57. Peplow, M.; Nat. Rev. Drug Discov. 2017, 16, 815 
58. Sohlenius-Sternbeck, A.-K.; Janson, J.; Bylund, J.; Baranczewski, P.; Breitholtz-Emanuelsson, A.; Hu, Y.; Tsoi, C.; Lindgren, A.; Gissberg, O.; Bueters, T.; Briem, S.; Juric, S.; Johansson, J.; Bergh, M.; Hoogstraate, J.; Curr. Drug Metab. 2016, 17, 253.

59. Wang, L.; Wu, Y.; Deng, Y.; Kim, B.; Pierce, L.; Krilov, G.; Lupyan, D.; Robinson, S.; Dahlgren, M. K.; Greenwood, J.; Romero, D. L.; Masse, C.; Knight, J. L.; Steinbrecher, T.; Beuming, T.; Damm, W.; Harder, E.; Sherman, W.; Brewer, M.; Wester, R.; Murcko, M.; Frye, L.; Farid, R.;
Lin, T.; Mobley, D. L.; Jorgensen, W. L.; Berne, B. J.; Friesner, R. A.; Abel, R.; J. Am. Chem. Soc. 2015, 137, 2695.

60. WEF World Economic Forum https://www.weforum.org/ agenda/2016/01/the-fourth-industrial-revolution-what-it-means-andhow-to-respond/, acessada em dezembro de 2017.

61. Amaral, A. T.; Andrade, C. H.; Kummerle, A.; Guido, R. V. C.; Quim. Nova 2017, 40, 694. 